Yin You Shareholder of: Johnson and Johnson, Employee of: Janssen Research \& Development, LLC, Yaung-Kaung Shen Shareholder of: Johnson and Johnson, Employee of: Janssen Research \& Development, LLC, Andrew Blauvelt Speakers bureau: AbbVie, Consultant of: AbbVie, Aclaris, Almirall, Arena, Athenex, Boehringer Ingelheim, Bristol-Myers Squibb, Dermavant, Dermira, Eli Lilly, FLX Bio, Forte, Galderma, Janssen, Leo, Novartis, Ortho, Pfizer, Regeneron, Sandoz, Sanofi Genzyme, Sun Pharma, and UCB Pharma DOI: 10.1136/annrheumdis-2021-eular.960

\section{AB0533 COMBINATION OF METHOTREXATE AND LEFLUNOMIDE IS SAFE AND HAS GOOD DRUG RETENTION AMONG PATIENTS WITH PSORIATIC ARTHRITIS}

M. Haroon ${ }^{1}$, S. Batool ${ }^{2}$, S. Asif ${ }^{2}$, F. Hashmi ${ }^{2}$, S. Ullah ${ }^{2}{ }^{1}$ Fatima Memorial Hospital \& FMH College of Medicine and Dentistry, Department of Rheumatology, Lahore, Pakistan; ${ }^{2}$ Fatima Memorial Hospital \& FMH College of Medicine and Dentistry, Department of Rheumatology, Lahore, Pakistan

Background: Among patients with psoriatic arthritis (PsA), there remains a considerable confusion regarding the effectiveness of conventional synthetic DMARDs (csDMARDs), especially methotrexate (MTX). The availability of biologic DMARDs and targeted synthetic DMARDs have revolutionised the management of psoriatic disease; however, it comes with a significant cost burden. We believe that combination of DMARDs, especially combining MTX and Leflunomide (LEF) provides a valuable low-cost treatment option for patients with PsA after failure of MTX monotherapy. Hence, in our practice, we are inclined to use combination of potent DMARDs after MTX failure, prior to considering biologic therapies. Little is known about the combination use of LEF and MTX in PSA, especially in the context of drug retention time and tolerability.

Objectives: We aimed to review our PsA cohort data especially examining the drug retention of first-line csDMARD monotherapy and combination csDMARDs.

Methods: In our centre, MTX is a preferred first line csDMARD, unless contraindicated, and patients are followed up with a protocol on 4-6 weekly basis unless complete remission is achieved. MTX if needed is escalated to the maximum tolerated dose (up to $25 \mathrm{mg} /$ week), and if PsA is still active then preferably LEF is added (usual starting dose for add-on therapy is $10 \mathrm{mg}$ a day and if needed escalated to $20 \mathrm{mg}$ a day, without any loading dose). Other csDMARDs, such as sulphasalazine are used, if needed. For this study, after written-informed consent, only those adult patients were included who had a follow up of at least 6 months with our rheumatology services, and were fulfilling CASPAR criteria. Moreover, only patients who were DMARD-naïve (no prior DMARD therapy for any cause, including psoriasis), and initiated DMARD as monotherapy after 1 April 2018 were included. If any patient had already been on any DMARDs prior to attending our rheumatology services was excluded.

Results: A total of 81 PsA patients [mean age $45.6 \pm 6$ years; $52 \%$ male; mean PsA disease duration $=9 \pm 4$ years; $35 \%$ with dactylitis, $42 \%$ with enthesitis, $17 \%$ with sacroiliitis, median current PASI $=2.6$, median number of swollen joints $=8.0$, median number of tender joints $=11.0]$ fulfilled the inclusion and exclusion criteria. As regards first-line csDMARD monotherapy, $88 \%(n=71)$ of patients were commenced on MTX. In total, $79 \%$ ( $n=56$ out of 71 ) of patients who were started on MTX as their first-line csDMARD therapy failed this monotherapy during follow-up ( $51=$ ineffective, 5 =intolerance). After a median follow-up of 22 months, MTX median drug retention among all MTX monotherapy users ( $n=71$ ) was only 7 months (IQR 5-7); and among MTX failures $(n=56)$, MTX monotherapy median drug retention was 6.0 months (IQR 4-8). Eighty percent ( $n=45$ out of 56) of the MTX monotherapy failure cohort was started on combination therapy of MTX and LEF (combo MTX+LEF); among them, only 7 patients needed escalation of therapy to bDMARDs, and the rest are still using combo MTX+LEF. It was noted that to date median drug retention time of combo MTX+LEF has been 8 months (IQR 7-11), and $84 \%$ ( $n=38$ out of 45 ) of these patients are still using this combo therapy. Significantly more patients managed to continue the combo MTX+LEF therapy compared to MTX monotherapy $(84 \%$ vs. $21 \%, p<0.001)$

Conclusion: Among csDMARD naïve PsA patients, $79 \%$ of patients failed MTX monotherapy with median drug retention time of only 6 months. Combination of MTX and LEF was well tolerated and had good drug retention time, with $84 \%$ of patients having ongoing treatment to date. Our data provides initial evidence that MTX and LEF combination therapy could be an effective treatment option for PsA

Disclosure of Interests: Muhammad Haroon Speakers bureau: Roche, Novartis, Grant/research support from: Abbvie, Pfizer, Shabnam Batool: None declared., Sadia Asif: None declared., Farzana Hashmi: None declared., Saadat Ullah: None declared.

DOI: 10.1136/annrheumdis-2021-eular.978

\section{AB0534 \\ THE IMPACT OF AXIAL INVOLVEMENT ON ACR RESPONSE IN PATIENTS WITH PSORIATIC ARTHRITIS RESULTS OF SUBANALYSIS OF THE PATERA STUDY}

T. Korotaeva ${ }^{1}$, I. Gaydukova ${ }^{2}$, V. Mazurov ${ }^{2}$, A. Samtsov ${ }^{3}$, V. Khayrutdinov ${ }^{3}$, A. Bakulev ${ }^{4}$, A. Kundzer ${ }^{5}$, N. Soroka ${ }^{6}$, A. Eremeeva ${ }^{7}{ }^{1}$ Nasonova Research Institute of Rheumatology, Laboratory of Spondyloarthritides and Psoriatic Arthritis, Moscow, Russian Federation; ${ }^{2}$ Mechnikov North-Western State Medical University, Department of Therapy and Rheumatology of Temporary Disability and Medical Care Quality Expertise, St-Petersburg, Russian Federation; ${ }^{3}$ Kirov Military Medical Academy, Department of Skin and Veneral Diseases, St-Petersburg, Russian Federation; ${ }^{4}$ Razumovsky Saratov State Medical University, Department of Dermatovenereology and Cosmetology, Saratov, Russian Federation; ${ }^{5}$ Belarusian Medical Academy of Postgraduate Education, Department of Cardiology and Rheumatology, Minsk, Belarus; ${ }^{6}$ Belarusian State Medical University, Department of Internal Diseases №2, Minsk, Belarus; ${ }^{7}$ JSC BIOCAD, Clinical Development Department, St-Petersburg, Russian Federation

Background: Inflammatory back pain (IBP) is a common symptom of axial disease in patients with psoriatic arthritis (PsA). The reported prevalence of axial disease in patients with PsA is quite variable and must be taken into account while choosing treatment strategy. Netakimab (NTK) is an anti-interleukin-17A monoclonal antibody approved for psoriasis, ankylosing spondylitis, PsA in Russia and Belarus.

Objectives: A subanalysis was aimed to investigate the ACR (American College of Rheumatology) 20/50/70 response rate in PsA patients with/without the axial disease, defined by the presence of IBP according to self-reported ASAS IBP criteria, 2009 at baseline.

Methods: PATERA is an ongoing phase 3 international double-blind, placebo-controlled clinical study (NCT03598751). 194 adult patients with PsA (CASPAR criteria, 2006) with inadequate response to csDMARD or one TNFi, were randomly assigned to receive NTK $120 \mathrm{mg}$ or placebo at weeks $0,1,2,4,6,8,10,14,18,22$. The ACR response was calculated in NTK-treated patients with IBP $(\operatorname{IBP}(+))$ and NTK-treated patients without IBP (IBP(-)) according to self-reported ASAS IBP criteria, 2009. Patients with missing values for categorical variables were considered as non-responders in the analysis.

Results: 97 PsA patients (N=54 IBP(+), N=43 IBP(-)) received NTK. Both subpopulations were comparable in gender, age, and PsA activity at baseline. There were no significant differences in ACR20 achievement between the groups (Figure 1). The percentage of patients with ACR50 was significantly $(p<0.05)$ higher in the IBP(-) subpopulation at weeks 4-20 (data not shown), but not at week 24 with $63 \% \mathrm{IBP}(+)$ and $79 \% \mathrm{IBP}(-)$ responders $(\mathrm{p} \geq 0.05)$. Similarly, IBP(+) patients had a lower frequency of ACR70 response (Figure 1).

Conclusion: NTK is effective in PsA treatment irrespectively of the presence of axial disease. Both IBP(-) and IBP(+) subpopulations achieved ACR20/50/70 as well, however, the benefit in IBP(-) patients was more pronounced.

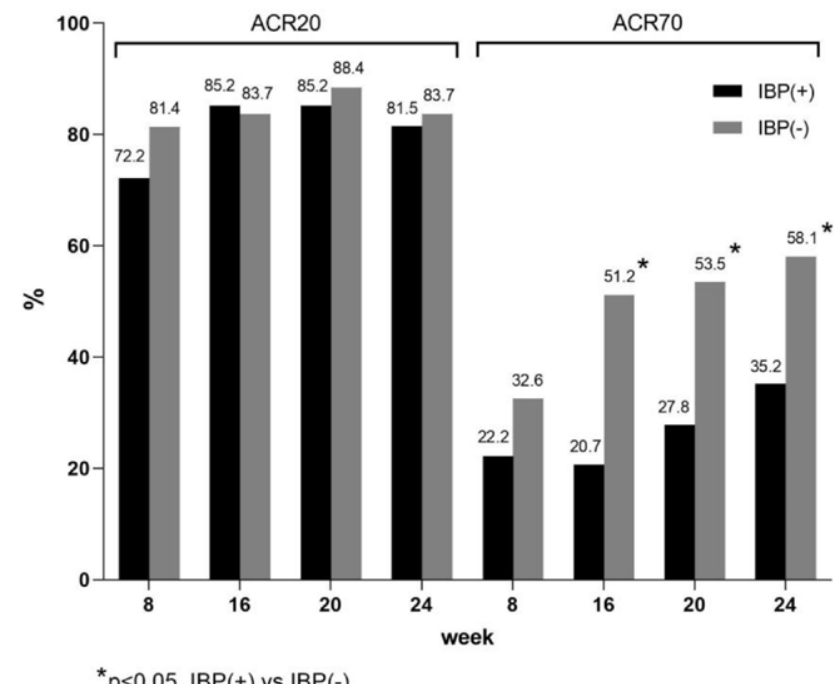

Figure $1 \mathrm{ACR}$ response rates

Acknowledgements: This study was sponsored by JSC BIOCAD.

Disclosure of Interests: Tatiana Korotaeva Speakers bureau: Abbvie, Biocad, Eli Lilly, Johnson \& Johnson, Janssen, Novartis, Pfizer, UCB, Inna Gaydukova 